\title{
Prognostic Significance of Chest Imaging by LUS and CT in COVID-19 Inpatients: The ECOVID Multicenter Study
}

\author{
Claudio Tana ${ }^{a}$ Fabrizio Riccib,c,d Maria Gabriella Coppola e Cesare Mantini ${ }^{b}$ \\ Fulvio Lauretani ${ }^{\mathrm{f}, \mathrm{g}}$ Daniele Campanozzi ${ }^{\mathrm{h}}$ Giulia Renda $^{\mathrm{b}}$ Sabina Gallinab Marina Lugaráe \\ Francesco Cipollone ${ }^{\mathrm{i}}$ Maria Adele Giamberardino ${ }^{\mathrm{j}} \quad$ Luciano Mucci $^{\mathrm{h}}$ \\ ${ }^{a}$ COVID-19 Medicine Unit and Geriatrics Clinic, SS Annunziata Hospital of Chieti, Chieti, Italy; ${ }^{b}$ Department of \\ Neuroscience, Imaging and Clinical Sciences, "G. d'Annunzio" University of Chieti-Pescara, Chieti, Italy; 'Department \\ of Clinical Sciences, Lund University, Malmö, Sweden; ${ }^{d}$ Casa di Cura Villa Serena, Città Sant'Angelo, Pescara, Italy; \\ eCOVID-19 Medicine Unit, Ospedale del Mare, Napoli, Italy; ' Department of Medicine and Surgery, University of \\ Parma, Parma, Italy; ${ }^{9}$ Cognitive and Motor Center, Geriatric-Rehabilitation Department of Parma, University-Hospital

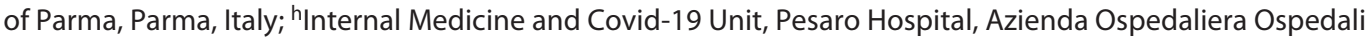 \\ Riuniti Marche Nord, Pesaro, Italy; 'COVID-19 Medicine Unit and Medical Clinic, SS Annunziata Hospital of Chieti, \\ Department of Medicine and Science of Aging, G D'Annunzio University of Chieti, Chieti, Italy; ${ }^{j}$ COVID-19 Medicine \\ Unit and Geriatrics Clinic, SS Annunziata Hospital of Chieti, Department of Medicine and Science of Aging, and \\ CAST, G D’Annunzio University of Chieti, Chieti, Italy
}

\section{Keywords}

Coronavirus disease 2019 · Lung ultrasound · Computed tomography $\cdot$ Lung ultrasound score $\cdot$ Mortality

\footnotetext{
Abstract

Background: Point-of-care lung ultrasound (LUS) score is a semiquantitative score of lung damage severity. High-resolution computed tomography (HRCT) is the gold standard method to evaluate the severity of lung involvement from the novel coronavirus disease (COVID-19). Few studies have investigated the clinical significance of LUS and HRCT scores in patients with COVID-19. Therefore, the aim of this study was to evaluate the prognostic yield of LUS and of HRCT in COVID-19 patients. Methods: We carried out a multicenter, retrospective study aimed at evaluating the prognostic yield of LUS and HRCT by exploring the survival curve of COVID-19 inpatients. LUS and chest CT scores were calculated retrospectively by 2 radiologists with $>10$ years of experience in
}

chest imaging, and the decisions were reached in consensus. LUS score was calculated on the basis of the presence or not of pleural line abnormalities, B-lines, and lung consolidations. The total score (range 0-36) was obtained from the sum of the highest scores obtained in each region. CT score was calculated for each of the 5 lobes considering the anatomical extension according to the percentage parenchymal involvement. The resulting overall global semiquantitative CT score was the sum of each single lobar score and ranged from 0 (no involvement) to 25 (maximum involvement). $\boldsymbol{R} \boldsymbol{e}$ sults: One hundred fifty-three COVID-19 inpatients (mean age $65 \pm 15$ years; 65\% M), including 23 (15\%) in-hospital deaths for any cause over a mean follow-up of 14 days were included. Mean LUS and CT scores were $19 \pm 12$ and $10 \pm 7$, respectively. A strong positive linear correlation between LUS and CT scores (Pearson correlation $r=0.754 ; R^{2}=0.568$; $p<0.001$ ) was observed. By ROC curve analysis, the optimal cut-point for mortality prediction was 20 for LUS score and 4.5 for chest CT score. According to Kaplan-Meier survival karger@karger.com www.karger.com/res (c) 2021 S. Karger AG, Base

Karger

Correspondence to:

Claudio Tana, claudio.tana@asl2abruzzo.it 
analysis, in-hospital mortality significantly increased among COVID-19 patients presenting with an LUS score $\geq 20$ (logrank 0.003; HR 9.87, 95\% Cl: 2.22-43.83) or a chest CT score $\geq 4.5$ (HR 4.34, 95\% Cl: 0.97-19.41). At multivariate Cox regression analysis, LUS score was the sole independent predictor of in-hospital mortality yielding an adjusted HR of 7.42 (95\% Cl: 1.59-34.5). Conclusion: LUS score is useful to stratify the risk in COVID-19 patients, predicting those that are at high risk of mortality.

(c) 2021 S. Karger AG, Basel

\section{Introduction}

At the end of December 2019, an anomalous cluster of pneumonia was identified in China. The causative agent was identified as a novel coronavirus (SARS- $\mathrm{CoV}-2$ ), and the outbreak has been declared as a public health emergency issue of international concern by the WHO. The rapid spread and high morbidity make this disease one of the most serious calamities of the last centuries [1].

The diagnosis of infection is made possible by the use of a nasopharyngeal swab, and high-resolution chest computed tomography (HRCT) is considered the gold standard to detect the damage and to evaluate the severity of lung involvement from the novel coronavirus disease (COVID-19) [2]. Different scores are being studied to evaluate the severity degree of lung involvement. The CT severity score proposed by Pan et al. [3] is useful to quantify the burden of pulmonary disease from COVID-19.

Point-of-care lung ultrasound (LUS) is emerging as a reliable tool to evaluate the damage extension from COVID-19 [4]. The LUS score is a semiquantitative score of lung damage severity based on the presence of findings such as pleural line abnormalities, B-lines, and lung consolidations. Few studies have hitherto investigated the clinical significance in terms of prognostic value of the LUS and HRCT scores in patients with COVID-19 [5]. Therefore, the aim of this study was to evaluate the prognostic yield of LUS and HRCT in COVID-19 patients, by calculating LUS and CT scores and by exploring the survival curve of COVID-19 inpatients.

\section{Methods}

Design of the Study

We carried out a multicenter, retrospective study aimed at evaluating the prognostic yield of LUS and HRCT in COVID-19 inpatients. The outcome was evaluated in all discharged patients versus those who died in the hospital. All medical records of patients with suspected SARS-CoV-2 infection and then confirmed by oropharyngeal swab and hospitalized in 2 medical departments in Italy, in the period March-May 2020, were analyzed. All the results regarding clinical and laboratory data, ultrasound tests, and pulmonary CT (performed in the first $48-72 \mathrm{~h}$ from the department admission) were analyzed retrospectively and aggregated anonymously.

\section{Study Population}

We included all consecutive COVID-19 inpatients aged $\geq 18$ years who underwent point-of-care LUS and chest CT on the same day between March 2020 and May 2020. Patients affected with active neoplasia were not deemed eligible.

Clinical information about symptoms, physical examination, major comorbidities, and cardiovascular risk factors was recorded. Furthermore, data about laboratory findings and positivity of oropharyngeal swab were acquired.

\section{Lung Ultrasound}

All patients underwent standard LUS using conventional ultrasound systems (Esaote MyLab ${ }^{\mathrm{TM}}$ Sigma and E-cube i7 Alpinion) with a convex low-frequency probe to scan lung parenchyma and a linear high-frequency probe to scan pleural area, in agreement with the standardized acquisition protocol [6] which provides the acquisition of 12 standard areas in patients able to maintain a sitting position (2 posterior, 2 lateral, and 2 anterior), based on standard sequence of evaluations using landmarks on chest anatomic lines.

In patients not able to maintain the sitting position and in patients on invasive ventilation, the posterior areas could be difficult to evaluate. In these cases, the operator will acquire a partial view of the posterior basal areas and start the echographic assessment from the lateral scanning areas on the midaxillary line below the internipple line.

The severity of lung findings was described numerically with the LUS score based on a reproducible and validated algorithm. LUS score was calculated retrospectively by 2 radiologists with $>10$ years of experience in chest imaging, and the decisions were reached in consensus. Each zone has been scored as follows:

- Score 0: the pleural line is continuous and regular and presence of A-lines (Fig. 1a1, a2);

- Score 1: the pleural line is indented and presence of multiple well-spaced B-lines (Fig. 1b2);

- Score 2: presence of coalescent B-lines with small to large subpleural consolidations (Fig. 1c1);

- Score 3: presence of dense and largely extended white lungs (Fig. 1c2) with or without lung consolidation.

The total score (range 0-36) was obtained from the sum of the highest scores obtained in each region.

\section{Chest CT}

Low-dose, unenhanced chest spiral, ungated CT scans for suspected COVID-19 were acquired on 2 different CT scanners: an 80 -slice system (Aquilion Prime; Canon Medical Systems Cooperation, Otowara, Japan) and a 128-slice system (Somatom Definition Edge; Siemens Healthineers, Erlangen, Germany). Scan parameters for Aquilion Prime included tube voltage $100 \mathrm{kV}$, automatic tube current (mAs) modulation, slice thickness $1 \mathrm{~mm}$, and slice increment $0.8 \mathrm{~mm}$. Scan parameters for Somatom Definition 

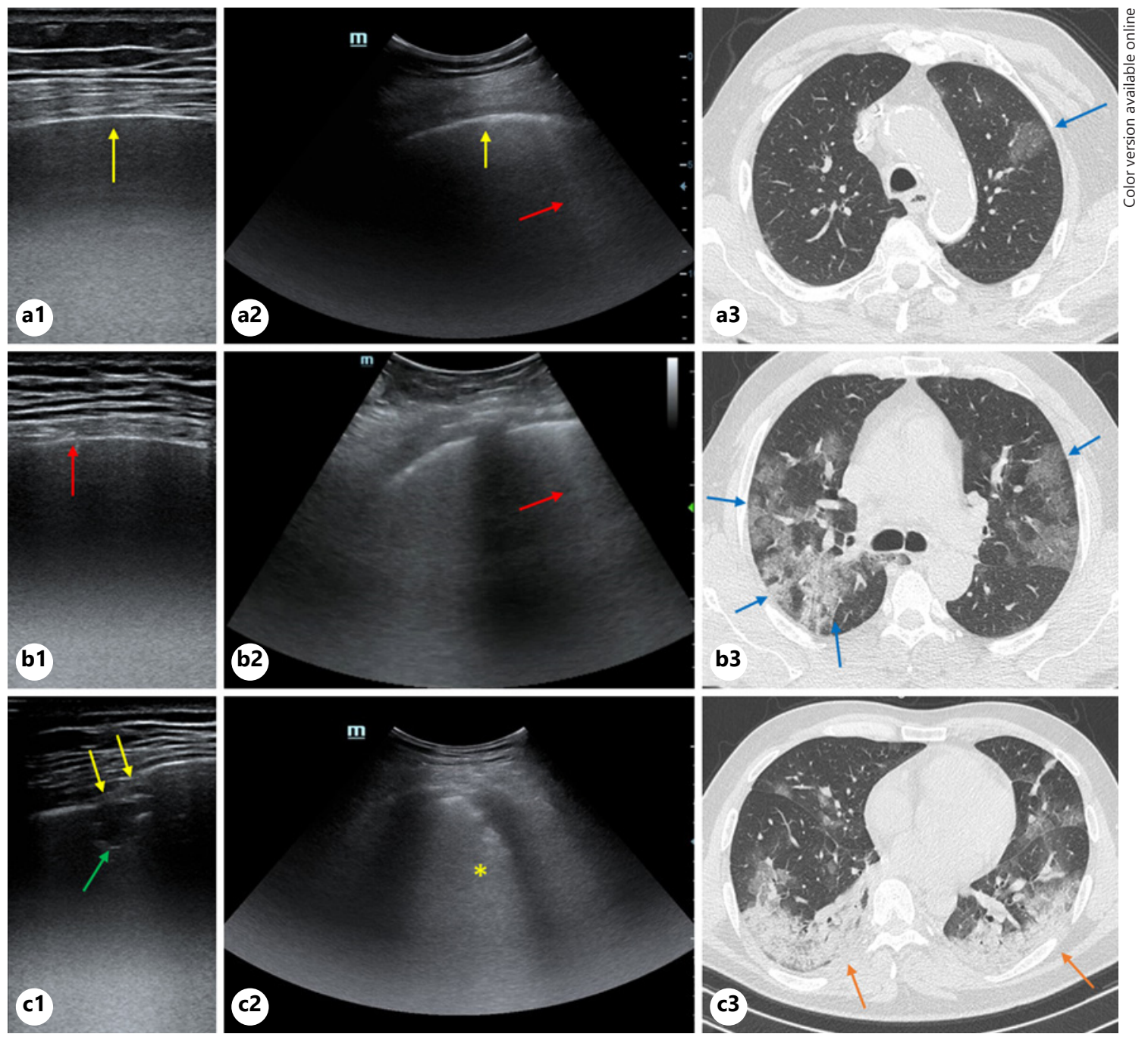

Fig. 1. Typical LUS findings obtained with linear (first column) and convex (second column) and corresponding axial unenhanced chest CT images (third column) of COVID-19 patients with various LUS scores. Lung images of a patient with a low LUS score (a); normal pleural line (yellow arrows) and focally visible B-line artifacts (red arrow) (a1, a2); corresponding lung CT scan showing focal area of ground-glass opacity (blue arrow) (a3). Lung images of a patient with a moderate LUS score (b); irregular pleural line and B-line (red arrows) (b1, b2); corresponding lung CT scan showing bilateral peripherally located ground-glass opacities (blue arrows) (b3). Lung images of a patient with a high LUS score (c); broken pleural line (yellow arrows), and below the breaking point, there is a subpleural consolidation (darker area) (green arrow) (c1); largely extended white lung $(*)$ (c2); corresponding lung CT scan showing large consolidated areas (orange arrows) (c3). LUS, lung ultrasound; CT, computed tomography.

Edge included tube voltage $120 \mathrm{Kv}$, automatic tube current (mAs) modulation, slice thickness $1 \mathrm{~mm}$, and slice increment $0.8 \mathrm{~mm}$.

All CT scans (from the lung apex to the costophrenic angle) were successfully acquired during a single inspiratory breath-hold, using the HRCT technique based on thin sections and high-spatial resolution kernel to improve lung parenchymal anatomical details. The standard glossary for thoracic imaging presented by the Fleischner Society and that defines abnormal radiological findings such as pulmonary consolidation, ground-glass opacity, and crazy-paving pattern was used [7]. The diagnosis of COVID-19 pneu- monia was based on the presence of consolidation (Fig. 1c3) ground-glass opacity (Fig. 1a3), and crazy-paving pattern (Fig. 1b3) according to previous studies $[8,9]$.

The CT severity score proposed by Pan et al. [3] was calculated for each of the 5 lobes considering the anatomical extension as follows: no involvement $=0 ;<5 \%$ involvement $=1 ; 5-25 \%$ involvement $=2 ; 26-50 \%$ involvement $=3 ; 51-75 \%$ involvement $=4$; and $>75 \%$ involvement $=5$. The resulting overall global semiquantitative CT score was the sum of each single lobar score and ranged from 0 (no involvement) to 25 (maximum involvement). Blinded 
Table 1. Demographic and clinical characteristics of the ECOVID population

\begin{tabular}{|c|c|c|c|c|}
\hline Covariate & Overall $(n=153)$ & Survivors $(n=130)$ & Deceased $(n=23)$ & $p$ value \\
\hline Age, years & $65 \pm 15$ & $64 \pm 15$ & $72 \pm 11$ & 0.014 \\
\hline Male sex, $n(\%)$ & $98(65)$ & $81(63)$ & $17(74)$ & 0.305 \\
\hline COVID-19 pneumonia & $124(80)$ & $101(77)$ & $23(100)$ & 0.012 \\
\hline Fever, $n(\%)$ & $111(72)$ & $91(70)$ & $20(87)$ & 0.093 \\
\hline Cough, $n(\%)$ & $112(73)$ & $91(70)$ & $21(93)$ & 0.033 \\
\hline GI tract symptoms, $n(\%)$ & $12(8)$ & $10(8)$ & $2(9)$ & 0.869 \\
\hline Dyspnea, $n(\%)$ & $107(69)$ & $85(65)$ & $22(96)$ & 0.004 \\
\hline Tachycardia, $n(\%)$ & $81(53)$ & $59(45)$ & $22(96)$ & $<0.001$ \\
\hline Hypertension, $n(\%)$ & $114(75)$ & $96(74)$ & $18(78)$ & 0.847 \\
\hline Diabetes, $n(\%)$ & $38(25)$ & $28(21)$ & $10(43)$ & 0.157 \\
\hline Atrial fibrillation, $n(\%)$ & $14(9)$ & $9(7)$ & $5(22)$ & 0.071 \\
\hline Prevalent CAD, $n(\%)$ & $40(27)$ & $26(20)$ & $14(61)$ & $<0.001$ \\
\hline Prevalent stroke, $n(\%)$ & $18(12)$ & $14(11)$ & $4(17)$ & 0.364 \\
\hline Dementia, $n(\%)$ & $36(23)$ & $26(20)$ & $9(39)$ & 0.044 \\
\hline COPD, $n(\%)$ & $61(40)$ & $45(35)$ & $16(70)$ & 0.002 \\
\hline Current smoker, $n(\%)$ & $60(39)$ & $48(37)$ & $11(48)$ & 0.394 \\
\hline Obesity, $n(\%)$ & $40(28)$ & $33(38)$ & $7(30)$ & 0.607 \\
\hline Chronic liver disease, $n(\%)$ & $20(13)$ & $16(12)$ & $4(17)$ & 0.911 \\
\hline $\mathrm{CKD}, n(\%)$ & $42(30)$ & $31(26)$ & $11(48)$ & 0.041 \\
\hline Creatinine, $\mathrm{mg} / \mathrm{dL}$ & $1.3 \pm 1.4$ & $1.3 \pm 1.4$ & $1.5 \pm 1.3$ & 0.432 \\
\hline $\mathrm{CRP}, \mathrm{mg} / \mathrm{L}$ & $7.4 \pm 7$ & $7.1 \pm 7.6$ & $8.9 \pm 5.9$ & 0.280 \\
\hline D-dimer, $\mathrm{ng} / \mathrm{mL}$ & $1.330(2,760)$ & $1,170(2,020)$ & $3,599(3,110)$ & 0.002 \\
\hline $\mathrm{LDH}, \mathrm{U} / \mathrm{L}$ & $314(218)$ & $285(188)$ & $378(284)$ & 0.014 \\
\hline LUS score & $19 \pm 12$ & $17 \pm 12$ & $27 \pm 6$ & $<0.001$ \\
\hline LUS score $\geq 20, n(\%)$ & $88(57)$ & $67(51)$ & $21(91)$ & $<0.001$ \\
\hline Chest CT score & $10 \pm 7$ & $10 \pm 7$ & $12 \pm 6$ & 0.465 \\
\hline Chest CT score $>4, n(\%)$ & $113(73)$ & $92(71)$ & $21(91)$ & 0.039 \\
\hline
\end{tabular}

$\mathrm{CA}$, coronary artery disease; $\mathrm{CKD}$, chronic kidney disease; COPD, chronic obstructive pulmonary disease; CRP, C-reactive protein; CT, computed tomography; LUS, lung ultrasound; GI, gastrointestinal.

to official reports, 2 radiologists with $>10$ years of experience in chest imaging retrospectively reviewed all the lung parenchymal CT scans, and the decisions were reached in consensus.

\section{Statistical Analysis}

The normality of distribution of the parameters was assessed by the Kolmogorov-Smirnov test. Continuous variables with normal distribution were described as mean $\pm \mathrm{SD}$ and nonnormal variables with median (interquartile range). Categorical variables were expressed as frequencies and percentages. For continuous values with normal distribution, comparisons between groups were made by the independent-samples $t$ test. Wilcoxon's signed rank test was applied for continuous values with nonnormal distribution. $\chi^{2}$ testing was performed for noncontinuous variables. One-way ANOVA test was used for group differences in continuous variables while Pearson's $\chi^{2}$ test was used for group differences in categorical variables. ROC curve analysis was performed in order to derive the optimal cut-point of LUS and CT scores for mortality prediction. Then, time-to-event analysis by Kaplan-Meier estimator and log-rank test was performed. The follow-up time was estimated as the time between hospital admission and date of in-hospital death or discharge. There were no missing values for survival status.

The ECOVID Study
The Schoenfeld residuals test was used to check the proportional hazards assumption. Hazard ratios (HR) with 95\% confidence intervals (CIs) were computed from Cox models to estimate the magnitude of the increased risk of death by LUS and CT scores. The selection of confounding variables for adjustment was based on the identification of risk factors for measured outcomes, further encompassing clinical meaningful variables due to biological plausibility to interact with the observed effect size estimates [10]. All multivariate models were therefore adjusted for the following potential confounders: age, gender, creatinine, and D-dimer. All calculations were performed using the Wizard 2 statistical software version 2.0.4 for Mac. All tests were 2-sided, and a $p$ value $<0.05$ was considered statistically significant.

\section{Results}

\section{ECOVID Study Population}

We collected complete clinical, radiological, and outcome information of 153 COVID-19 inpatients (mean age $65 \pm 15$ years; 65\% male sex) and recorded 23 (15\%) 
Fig. 2. Pearson's correlation analysis. Scatter diagram demonstrating a strong positive linear correlation between LUS and chest CT scores. LUS, lung ultrasound; CT, computed tomography.
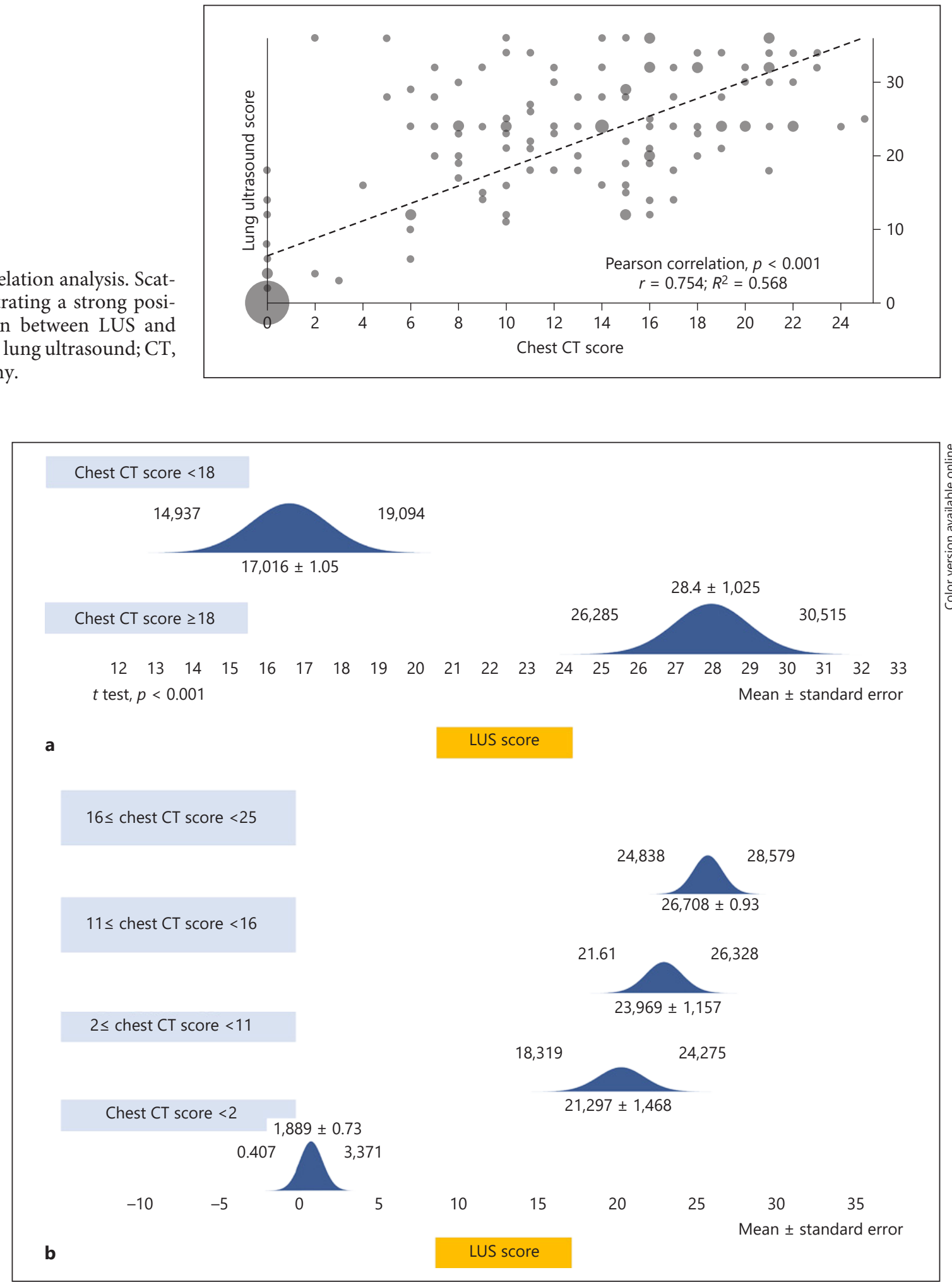

Fig. 3. LUS score by chest CT score subgroups. a LUS score was significantly higher in the subgroup of patients presenting with chest CT score $\geq 18$ compared with the subgroup with chest CT score $<18$. $\mathbf{b}$ LUS score by chest CT score quartiles. LUS score increased significantly across quartiles of chest CT score. LUS, lung ultrasound; CT, computed tomography. 


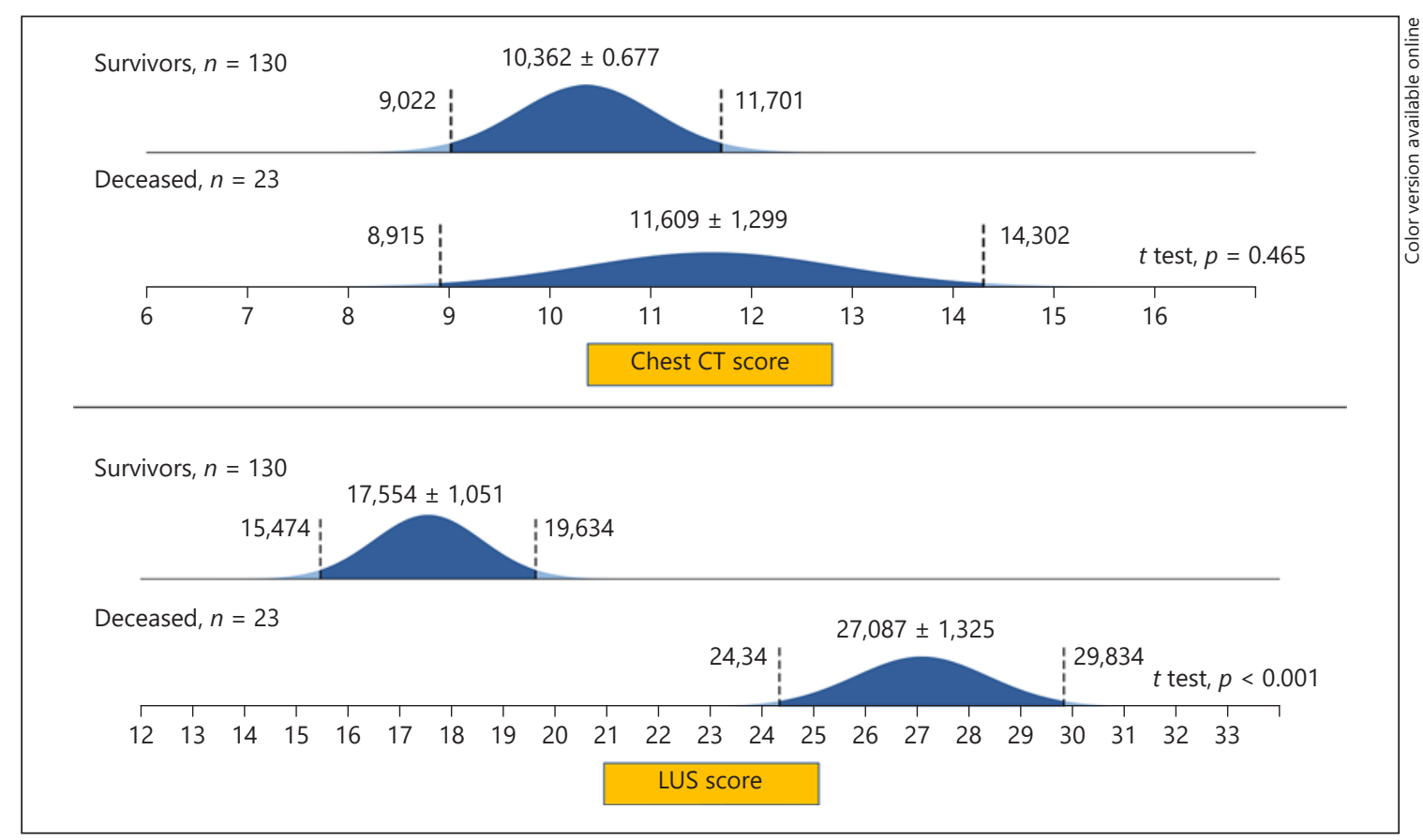

Fig. 4. Chest CT and LUS score by survivorship status. Chest CT score was similar between survivor and deceased COVID-19 inpatients. LUS score was significantly higher in deceased COVID-19 inpatients compared with survivors. LUS, lung ultrasound; CT, computed tomography.

in-hospital deaths for any cause over a mean follow-up of 14 days (range 1-32 days). Demographic and clinical characteristics of the ECOVID study cohort are summarized in Table 1. Compared with survivors, deceased patients were significantly older, had more often a history of coronary artery disease, dementia, COPD, and chronic kidney disease, presented more frequently with overt COVID-19 pneumonia, and were more symptomatic upon admission (tachycardia, cough, and dyspnea); deceased patients presented also with higher LDH and Ddimer levels.

\section{Chest Imaging}

All patients underwent LUS and chest CT on the same day. In our cohort, mean LUS score was $19 \pm 12$, and mean chest CT score was $10 \pm 7$. A strong positive linear correlation between LUS and CT scores (Pearson correlation $r=0.754 ; R^{2}=0.568 ; p<0.001$ ) was observed (Fig. 2). LUS score was significantly higher in the subgroup of patients presenting with chest CT score $\geq 18$ compared with the subgroup with chest CT score $<18$ and across quartiles of chest CT scores (Fig. 3). Compared with survivors, deceased patients had significantly higher LUS score (27 \pm 6 vs. $17 \pm 12 ; p<0.001)$ but similar chest CT score (12 \pm 6 vs. $10 \pm 7 ; p=0.465$ ) on admission (Fig. 4).

\section{Survival Analysis}

By ROC curve analysis, the optimal cut-point for mortality prediction was 20 for LUS score and 4.5 for chest CT score (see online suppl. Fig. 1; for all online suppl. material, see www.karger.com/doi/10.1159/000518516). According to Kaplan-Meier survival analysis, in-hospital mortality significantly increased among COVID-19 patients presenting with an LUS score $\geq 20$ (log-rank 0.003; HR 9.87, 95\% CI: 2.22-43.83) or a chest CT score $\geq 4.5$ (HR 4.34, 95\% CI: 0.97-19.41) (Fig. 5). At multivariate Cox regression analysis, LUS score was the sole independent predictor of in-hospital mortality yielding an adjusted HR of 7.42 (95\% CI: 1.59-34.5) (Fig. 5).

\section{Discussion}

In this study, we found that LUS score is a valuable tool not only to reveal the presence of and quantify the parenchymal damage from the SARS-CoV-2 infection but also for predicting clinical deterioration and mortality in $\mathrm{CO}-$ VID-19 in patients. COVID-19 pneumonia can show a plethora of ultrasound abnormalities, such as B-lines, pleural line abnormalities, significant consolidations and bilateral involvement, expression of deep parenchymal dam- 


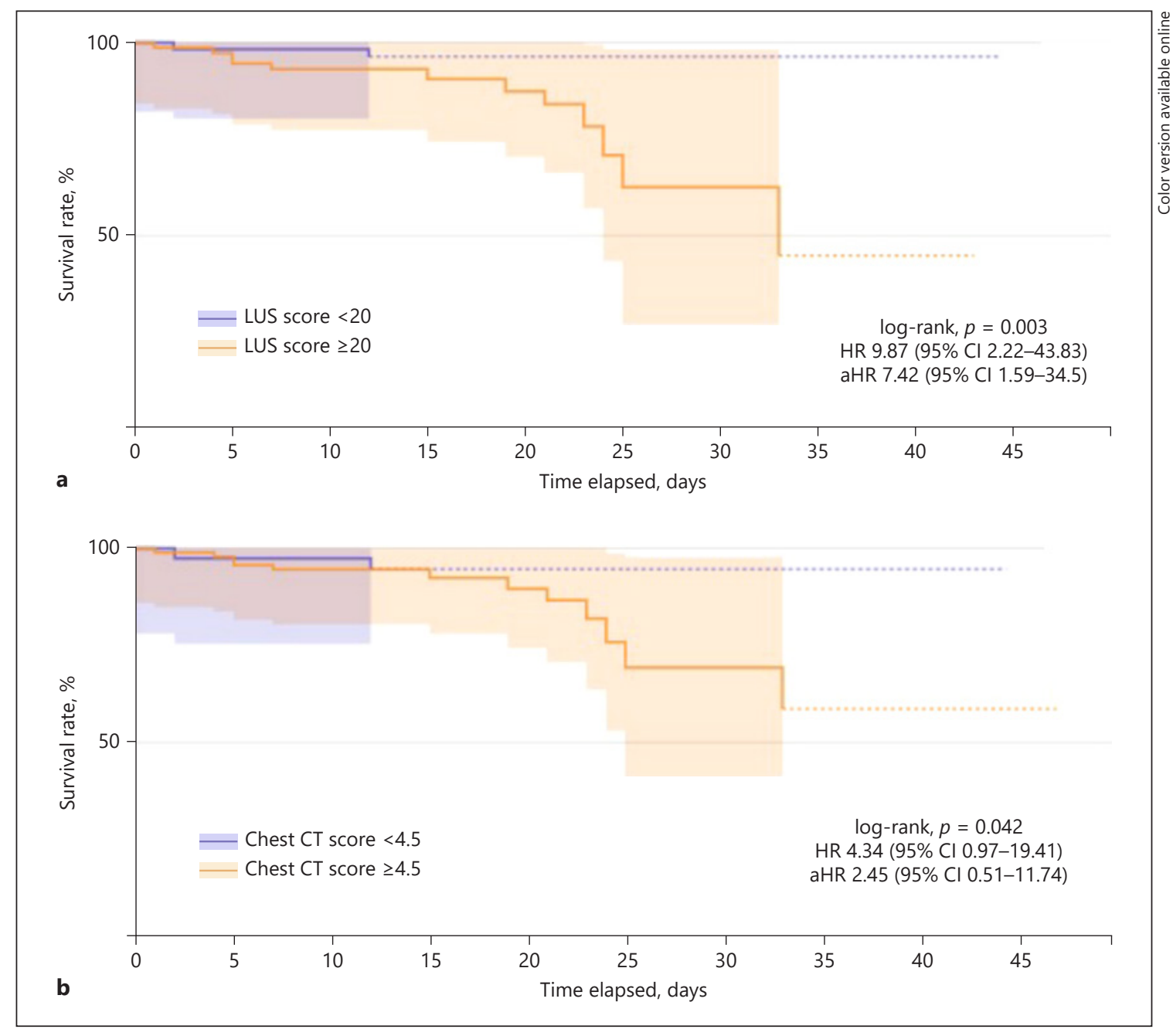

Fig. 5. Kaplan-Meier survival analysis. a Kaplan-Meier survival curve by levels of LUS score. LUS score $>20$ upon admission was associated with significantly higher in-hospital mortality. b KM curve by levels of CT score. Chest CT score $>4.5$ upon admission was associated with significantly higher in-hospital mortality. Percentage of survival is expressed on the $y$-axis, while time elapsed (days) of the observation period is expressed on the $x$-axis. HR, hazard ratio; aHR, hazard ratio adjusted for age, sex, D-dimer, and renal function; LUS, lung ultrasound; CT, computed tomography.

age, and impairment $[11,12]$. Beyond the LUS usefulness in terms of diagnostic yield, LUS seems to be effective also to predict the patient's outcome. Few studies have investigated the prognostic significance of LUS score in COVID-19 patients so far. Recently, LUS score has demonstrated to be useful for predicting adverse outcomes, ICU admission, and endotracheal intubation in COVID-19 patients that manifest overt parenchymal alterations [13].

Lichter et al. [14] have recently found that pathological LUS findings are associated with the need for noninvasive mechanic ventilation and clinical deterioration in terms of risk of loss of aeration in anterior lung segments, for a total LUS score $>18$, that was the optimal cutoff point compared to a lower LUS score ranging between 0 and 18 . However, prediction accuracy for this cutoff was lower (sensitivity $=62 \%$ ) [14]. A cutoff of 20 seems to be more sensitive to predict the in-hospital mortality from COVID-19 pneumonia. Such cutoff could be useful to predict the risk of clinical deterioration to those presenting a significant quote of parenchymal damage that can lead to adverse outcome and death. Such data are in line with the recent findings of Tombini et al. [12], who found that a cutoff of 20 significantly predicted adverse events such as endotracheal intubation and adverse outcomes. 
In our study, the finding of a positive and linear correlation between LUS and chest CT score confirms that both parameters can be useful to quantify the parenchymal impairment with accuracy and predict adverse outcomes in COVID-19 patients, where a significant increase in LUS score can be shown between groups with chest CT score $\geq 18$ versus $<18$ and across quartiles. Previously, Nouvenne et al. [15]demonstrated a significant correlation between LUS and a different visual CT scoring in a smaller sample size $(r=0.65, p<0.001)$. In this study, we used the score suggested by Pan et al. [3] that is based on a percentage tissue involvement, in order to try to define more accurately the parenchymal impairment from the SARS-CoV-2 infection.

Zhu et al. [16] reported a good correlation between LUS and chest CT score $(r=0.82, p<0.001)$ in a small population of COVID-19 patients with pneumonia of different severity degree and a significant negative correlation with the respiratory rate oxygenation index on the first $(r=-0.85, p<0.001)$ versus second and third week after symptom onset $(r=-0.71, p<0.001$, and $r=-0.76$, $p<0.001$, respectively), confirming the usefulness of LUS in assessing severity of COVID-19 pneumonia both in critically and noncritically ill patients [16]. The diagnostic efficacy of bedside US is significantly high for patients with severe form of the disease. LUS score is particularly important for the noninvasive assessment and dynamic observation of lung lesions [17].

The recent report of Dargent et al. [18] has found a good capability of LUS score to follow the parenchymal damage over time and to monitor the pneumonia evolution. Increasing LUS score values were associated with evolution to refractory hypoxemia, ventilator-associated pneumonia, and intubation and vice versa LUS score decreased in extubated patients, with a good agreement between CT scans and LUS. Although the sample size was small, these data have first suggested the importance of LUS to monitor the pneumonia evolution during the hospital stay, avoiding unnecessary further HRCT examination and exposure to radiation because LUS can be easily repeated at patient's bed, without the need to move the patient from one department to another [18].

In our study, we found that the LUS score has a significant predictive value of in-hospital mortality also at a multivariate analysis. LUS score was indeed significantly higher in deceased COVID-19 inpatients as compared with survivors, differently from the chest CT score that was similar between the 2 groups. Previously, chest CT score proved useful to predict patient's mortality in the short-term follow-up. Francone et al. [19] have demon- strated also a positive correlation with clinical parameters such as age, inflammatory biomarkers, and severity of clinical categories.

It is widely debated which method should be employed as the first-line exam in the routine evaluation of COVID-19 patients. Chest CT is largely accepted as the firstline method in most COVID-19 hospitals for evaluating patients presenting with respiratory failure at the symptom onset because it can be useful to quantify the parenchymal damage at the time zero and to give first indications for the patient's treatment. Furthermore, it is employed to monitor the evolution of lung damage from admission to recovery [3]. However, unlike ultrasound, HRCT involves a greater expenditure in terms of time, resources, need of sanitizing machines, and to move unstable patients from the clinical to the radiological setting for the image acquisition, with a consequent increased risk of infection [20]. LUS has several advantages compared to HRCT such as low cost, no radiation exposure, rapidity of image acquisition, and execution at the patient's point of care, all factors that are important in some conditions of urgency such as clinical and/or respiratory deterioration which it makes necessary the use of a noninvasive or (in worst cases) invasive respiratory support [21-23].

\section{Strengths and Limitations}

Our study has strengths and limitations. Strengths are (i) the robustness of the data obtained from a large, reallife, adult general population, where ill COVID-19 individuals with pneumonia were included; (ii) the reliability of collected data from both US and CT imaging in all patients. Limitations include the risk of inclusion bias and residual confounding, the lack of adjustment for all potential confounders, and the lack of important pieces of information such as respiratory exchanges measured with fractional inspired oxygen concentration and respiratory support. Further, we were not able to weigh the burden of comorbidities of our population, for example, by the ageadjusted Charlson Comorbidity Index, as such an index requires baseline information that was not available in our multicenter dataset. Finally, bearing in mind the realworld emergency setting and the lack of robust and reliable data from the previous literature to calculate the sample size at intended power, our study was based on a convenience sample size; hence, our findings should be interpreted as hypothesis generating. It is also important considering that our results are linked to the prevalence of the disease, which was particularly high during the first wave of the pandemic; therefore, with lower prevalence, the significance of our analysis needs to be revisited. 


\section{Conclusions}

LUS is a low-cost, fast, and noninvasive alternative to HRCT both for the first-line evaluation of COVID-19 patients and for monitoring the evolution of lung damage, avoiding the patient's exposure to repeated radiations with optimization of patient evaluation and follow-up. LUS score can be useful for stratification of the risk of death in COVID-19 patients, predicting those who can be at the highest risk of mortality and therefore in need of active and closer surveillance. Future studies could be addressed to the outpatient setting because LUS could be of particular utility in the primary care setting due to the HRCT unavailability and to screen those patients who are at the highest risk of deterioration, who could benefit from an early hospital admission.

\section{Statement of Ethics}

The ECOVID study protocol was approved by the Institutional Ethics Committee of Ancona (ID 2020144, May 28, 2020) and from the satellite centers involved in data collection. The study was conducted in accordance with the principles of the Declaration of Helsinki. Due to the retrospective design of the study and anonymous collection of data, informed consent signature was waived, in compliance with the Italian law, except in the cases where it was possible to give adequate information to patients, for example, for patients referring to the medical department for clinical follow-up after discharge.

\section{Conflict of Interest Statement}

The authors have no conflicts of interest to declare.

\section{Funding Sources}

No funding supported the study.

\section{Author Contributions}

C.T. and L.M. designed the study. L.M., M.G.C., D.C., and M.L. conducted the study and collected data. F.R. and F.L. analyzed the data. C.T. and F.R. drafted the manuscript. C.T., F.R., C.M., G.R., S.G., F.C., and M.A.G. gave support in the conduction of the study and revised the manuscript for important intellectual content.

\section{Data Availability Statement}

The data that support the findings of this study are available on request from the corresponding author. The data are not publicly available due to privacy/ethical restrictions.

\section{References}

1 Polverino F, Stern DA, Ruocco G, Balestro E, Bassetti M, Candelli M, et al.; ItaliCO Study Group. Comorbidities, cardiovascular therapies, and COVID-19 mortality: a Nationwide, Italian Observational Study (ItaliCO). Front Cardiovasc Med. 2020 Oct 9;7:585866.

2 Colombi D, Petrini M, Maffi G, Villani GD, Bodini FC, Morelli N, et al. Comparison of admission chest computed tomography and lung ultrasound performance for diagnosis of COVID-19 pneumonia in populations with different disease prevalence. Eur J Radiol. 2020 Dec;133:109344.

3 Pan F, Ye T, Sun P, Gui S, Liang B, Li L, et al. Time course of lung changes at chest CT during recovery from coronavirus disease 2019 (COVID-19). Radiology. 2020;295(3):715-21.

4 Ji L, Cao C, Gao Y, Zhang W, Xie Y, Duan Y, et al. Prognostic value of bedside lung ultrasound score in patients with COVID-19. Crit Care. 2020 Dec 22;24(1):700. PMID: 33353548; PMCID: PMC7754180.

5 De Alencar JCG, Marchini JFM, Marino LO, da Costa Ribeiro SC, Bueno CG, da Cunha $\mathrm{VP}$, et al. Lung ultrasound score predicts outcomes in COVID-19 patients admitted to the emergency department. Ann Intensive Care. 2021 Jan 11;11(1):6.
6 Mojoli F, Bouhemad B, Mongodi S, Lichtenstein D. Lung ultrasound for critically ill patients. Am J Respir Crit Care Med. 2019;199(6):701-14.

7 Hansell DM, Bankier AA, MacMahon H, McLoud TC, Müller NL, Remy J. Fleischner society: glossary of terms for thoracic imaging. Radiology. 2008;246:697-722.

8 YeZ, Zhang Y, Wang Y, Huang Z, Song B. Chest CT manifestations of new coronavirus disease 2019 (COVID-19): a pictorial review. Eur Radiol. 2020 Aug;30(8):4381-9. Epub 2020 Mar 19. PMID: 32193638; PMCID: PMC7088323.

9 Salehi S, Abedi A, Balakrishnan S, Gholamrezanezhad A. Coronavirus disease 2019 (COVID-19): a systematic review of imaging findings in 919 patients. AJR Am J Roentgenol. 2020;215:87-93.

10 Groenwold RH, Klungel OH, Grobbee DE, Hoes AW. Selection of confounding variables should not be based on observed associations with exposure. EurJ Epidemiol. 2011;26:589-93.

11 Kong S, Wang J, Li Y, Tian Y, Yu C, Zhang D, et al. Value of bedside lung ultrasound in severe and critical COVID-19 pneumonia. Respir Care. 2021 Mar 23;66(6):920-7.

12 Tombini V, Di Capua M, Capsoni N, Lazzati A, Bergamaschi M, Gheda S, et al. Risk stratification in COVID-19 pneumonia - deter- mining the role of lung ultrasound. Ultraschall Med. 2021 Feb 18. Epub ahead of print. English. PMID: 33601427.

13 Ji L, Cao C, Gao Y, Zhang W, Xie Y, Duan Y, et al. Prognostic value of bedside lung ultrasound score in patients with COVID-19. Crit Care. 2020 Dec 22;24(1):700.

14 Lichter Y, Topilsky Y, Taieb P, Banai A, Hochstadt A, Merdler I, et al. Lung ultrasound predicts clinical course and outcomes in COVID-19 patients. Intensive Care Med. 2020;46:1873-83.

15 Nouvenne A, Zani MD, Milanese G, Parise A, Baciarello M, Bignami EG, et al. Lung ultrasound in COVID-19 pneumonia: correlations with chest $\mathrm{CT}$ on hospital admission. Respiration. 2020;99(7):617-24.

16 Zhu F, Zhao X, Wang T, Wang Z, Guo F, Xue $\mathrm{H}$, et al. Ultrasonic characteristics and severity assessment of lung ultrasound in COVID-19 pneumonia in Wuhan, China: a retrospective, Observational Study. Engineering. 2021;7(3):367-75.

17 Lu W, Zhang S, Chen B, Chen J, Xian J, Lin Y, et al. A clinical study of noninvasive assessment of lung lesions in patients with coronavirus disease-19 (COVID-19) by bedside ultrasound. Ultraschall Med. 2020;41(3):300-7. 
18 Dargent A, Chatelain E, Kreitmann L, Quenot JP, Cour M, Argaud L; COVID-LUS Study Group. Lung ultrasound score to monitor COVID-19 pneumonia progression in patients with ARDS. PLoS One. 2020 Jul 21; 15(7): $\mathrm{e} 0236312$.

19 Francone M, Iafrate F, Masci GM, Coco S, Cilia F, Manganaro L, et al. Chest CT score in COVID-19 patients: correlation with disease severity and short-term prognosis. Eur Radiol. 2020;30(12):6808-17.
20 Smith MJ, Hayward SA, Innes SM, Miller ASC. Point-of-care lung ultrasound in patients with COVID-19 - a narrative review. Anaesthesia. 2020;75(8):1096-104.

21 Allinovi M, Parise A, Giacalone M, Amerio A, Delsante $\mathrm{M}$, Odone A, et al. Lung ultrasound may support diagnosis and monitoring of COVID-19 pneumonia. Ultrasound Med Biol. 2020;46(11):2908-17.
22 Moore S, Gardiner E. Point of care and intensive care lung ultrasound: a reference guide for practitioners during COVID-19. Radiography. 2020;26(4):e297-302.

23 Shaw JA, Louw EH, Koegelenberg CFN. Lung ultrasound in COVID-19: not novel, but necessary. Respiration. 2020;99(7):545-7. Epub 2020 Jul 13. PMID: 32659765; PMCID: PMC7445384. 